\title{
Utilization of Histidine by Caulobacter crescentus
}

\author{
By DONNA M. FERBER, FARUKH KHAMBATY AND BERT ELY* \\ Department of Biology, University of South Carolina, Columbia, South Carolina 29208, USA
}

(Received 23 March 1988)

\begin{abstract}
Caulobacter crescentus has an inducible pathway which is responsible for the degradation of histidine. Induction of this pathway occurs in the presence of both glucose and ammonia. Growth yield experiments indicate that only two of the three available nitrogens are used for growth suggesting that formamide may be produced as a waste product. However, formamide was not detected in the culture fluid and formate was formed instead. These results suggest that histidine may be degraded in a novel pathway which results in the production of 1 mol each of ammonia, glutamate and formate per mol of histidine. The third nitrogen from histidine appears to be sequestered in some kind of secondary metabolite.
\end{abstract}

\section{INTRODUCTION}

Caulobacter crescentus is a Gram-negative, prosthecate bacterium which has evolved to occupy ecological niches characterized by low nutrient content (Poindexter, 1964). While there have been some studies of Caulobacter nutrition, most research has been focused on the dimorphic nature of the cell cycle. Currently, biochemical and genetic studies are underway in several laboratories to determine how various genes are regulated in a cell-cycle-dependent fashion. In order to provide a basis for comparison, we have begun to study several catabolic genes whose expression is likely to be regulated independently of the cell cycle. Relatively few studies have been conducted concerning genes involved in carbon or nitrogen metabolism in C. crescentus. There has been some indication that the expression of lactose-degrading enzymes may be subject to catabolite repression by glucose (Kurn et al., 1977), and it has been established that guanosine 5'-diphosphate $3^{\prime}$-diphosphate is involved in the response to nitrogen starvation (Chiaverotti et al. 1981). We have chosen to study the expression of the enzymes responsible for histidine catabolism since this amino acid can serve as a nitrogen source for $C$. crescentus and since the catabolism of histidine by micro-organisms has been extensively studied.

In the enteric bacteria that can metabolize histidine (Magasanik \& Bowser, 1955; Meiss et al., 1969), and in the Gram-positive bacterium Bacillus subtilis (Chasin \& Magasanik, 1968), $1 \mathrm{~mol}$ of histidine is degraded via a four-enzyme pathway to 1 mol each of ammonia, formamide and glutamate (Fig. 1). In contrast, the four Pseudomonas species studied utilize a five-enzyme pathway to yield formate, glutamate and 2 mols of ammonia per mol of histidine degraded (Coote \& Hassall, 1973a, b; Leidgh \& Wheelis, 1973; Lessie \& Neidhardt, 1967; Tabor, 1955). In either pathway, the first three steps are the same. Histidase (EC 4.3.1.3) converts histidine to urocanate with the release of ammonia then the urocanate is converted to imidazolone propionate and then to $N$-formimino-L-glutamate (FIGLU) by urocanase (EC 4.2.1.49) and imidazolone propionase (EC 3.5.2.7), respectively. In the four-enzyme pathway, FIGLU is converted to glutamate and formamide by formiminoglutamase (EC 3.5.3.8), while in the fiveenzyme pathway FIGLU is converted by formiminoglutamate iminohydrolase (EC 3.5.3.13) to formylglutamate and ammonia. The formylglutamate is then converted to glutamate and formate by formylglutamate hydrolase.

Abbreviations: FIGLU, $N$-formimino-L-glutamate; CTAB, cetylhexadecyltrimethylammonium bromide. 


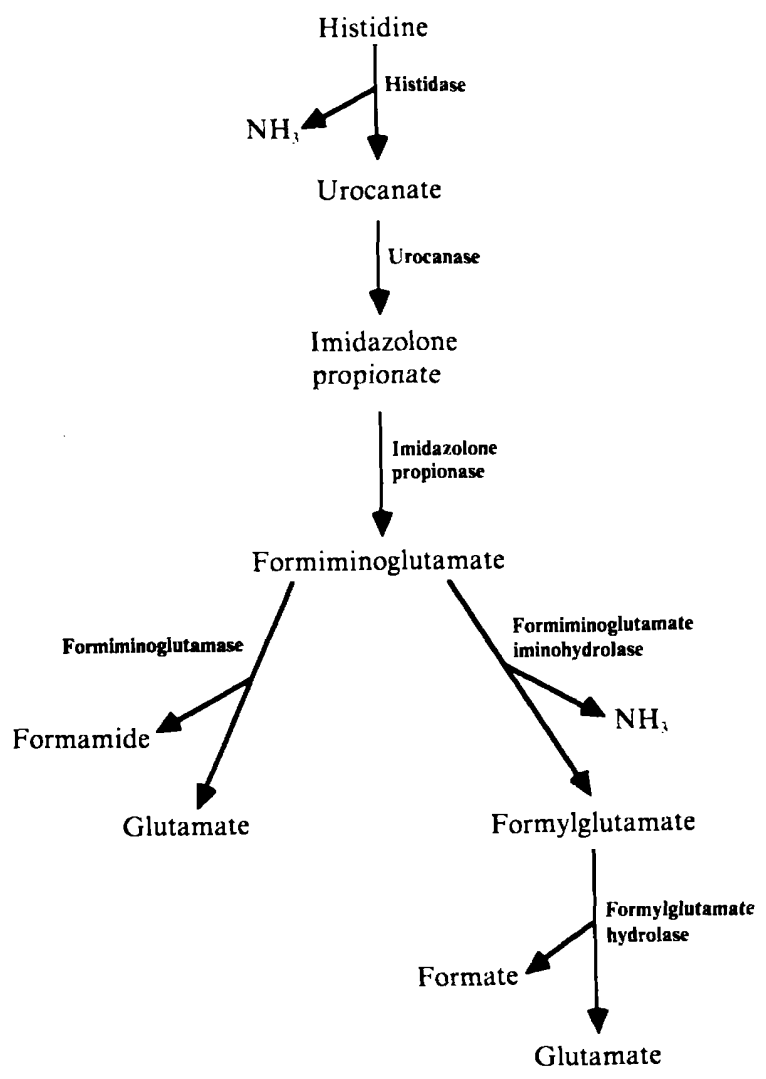

Fig. 1. Pathways of histidine degradation.

In C. crescentus, histidine can serve as the sole source of nitrogen even though histidine causes a transient growth inhibition when added to a minimal medium (Ferber \& Ely, 1982). Furthermore, growth experiments with mutants unable to assimilate ammonia indicated that histidine could be converted to glutamate (Ely et al., 1978).

\section{METHODS}

Bacterial strains and growth media. The $C$. crescentus wild-type strain CB15 used in these studies was originally described by Poindexter (1964). The minimal salts medium was the M2 medium described by Johnson \& Ely (1977) with $\mathrm{NH}_{4} \mathrm{Cl}$ omitted. For growth-yield experiments, cultures were aerated and incubated for $2 \mathrm{~d}$ at $33^{\circ} \mathrm{C}$. Pseudomonas putida strain PRS-1 was obtained from Dr Allen Phillips and growth-yield experiments were done in M9 medium (Davis et al., 1980) with $\mathrm{NH}_{4} \mathrm{Cl}$ omitted.

Enzyme assays. Histidase activity was measured as described by Smith \& Magasanik (1971) except that the pH optimum for C. crescentus histidase was 9.8. Urocanase was assayed as described by Smith $e$ t al. (1971) and formiminoglutamate iminohydrolase was assayed as described by Lund \& Magasanik (1965). Total protein was measured using the method of Lowry with bovine serum albumin for a standard. For whole cell preparations alkali solubilization preceded the Lowry assay. Histidine uptake experiments were done as described by Ferber \& Ely (1982).

\section{RESULTS AND DISCUSSION}

\section{Utilization of histidine as a nitrogen source}

In order to determine which intermediates of the histidine degradative pathways wild-type C. crescentus could take up and metabolize, we spread samples of washed cells on agar plates containing defined media lacking a nitrogen source. Small amounts of each test compound were 
spotted on the plates and growth stimulation was examined after 48 or $96 \mathrm{~h}$. Of the compounds tested, histidine, urocanate, formyl-L-glutamate and glutamate stimulated growth suggesting that they could serve as nitrogen sources. Formamide, formimino-L-glutamate, histidinol and D-histidine did not stimulate growth. Experiments in liquid medium indicated that histidine at concentrations from 1 to $10 \mathrm{~mm}$ supported growth with doubling times of 5 to $5.5 \mathrm{~h}$.

Histidine was tested for the ability to serve as the sole source of carbon for $C$. crescentus wildtype strain CB15 and was unable to support growth. Since histidine can be degraded to glutamate (Ely et al., 1978), and since glutamate can serve as the sole source of carbon for C. crescentus (Ferber \& Ely, 1982), it was surprising that histidine could not serve as the sole source of carbon as well. One explanation could be that histidine, as well as a number of other amino acids, causes growth inhibition just as it does in the presence of glucose and ammonia (Ferber \& Ely, 1982). In the presence of glucose this inhibition is transient and growth resumes as the histidine is degraded. Thus histidine might be used as a carbon source in the presence of an additional carbon source which could enable the histidine to be metabolized and detoxified. In order to test this hypothesis, we grew cultures in the presence of excess $\mathrm{NH}_{4} \mathrm{Cl}$ and limiting amounts of glucose and with varying amounts of histidine. Under these conditions, the histidine was utilized as a source of carbon and energy. Growth yields in the presence of $1 \mathrm{~mm}$-glucose and $1 \mathrm{~mm}$-histidine were approximately twice those observed with the glucose alone (data not shown). Thus, it appears that histidine can be used as a carbon source if the cells can grow on an alternative carbon source long enough to convert the histidine to overcome the initial growth inhibition.

\section{Growth yields with limiting amounts of histidine as a nitrogen source}

Depending on the nature of the histidine utilization pathway, either 2 or $3 \mathrm{mols}$ of nitrogen are made available for cellular metabolism. In order to make inferences about the pathway utilized by $C$. crescentus, we measured the growth yield after incubation in minimal medium containing limiting amounts of histidine as the sole source of nitrogen. Growth yields were compared to those obtained from experiments using limiting amounts of $\mathrm{NH}_{4} \mathrm{Cl}$, and indicated that only 2 mols of assimilable nitrogen are produced for each mol of histidine degraded (Table 1). When cultures were examined by phase-contrast microscopy after growth in limiting ammonia or histidine, cell size and shape appeared normal although they did appear to contain some granular deposits. However, in contrast to the undocumented observation of Chiaverotti $e t$ al. (1981), no swarmer cells were present and the majority of cells appeared to be predivisional cells with stalks of normal length. Therefore, cells seem to accumulate at the predivisional stage, and stalk elongation is not enhanced during nitrogen limitation as it is during phosphate limitation. Similar experiments using urocanate as a nitrogen source could not be interpreted since growth was much slower and the resulting cells were elongated and twisted. These results suggested that histidine might be degraded in a four-enzyme pathway to ammonia, glutamate and formamide, since this pathway would produce only 2 usable mols of nitrogen per mol of histidine. Control experiments using the same stock solutions of $\mathrm{NH}_{4} \mathrm{Cl}$ and histidine were done with Pseudomonas putida strain PRS-1. Growth yields with $0.5 \mathrm{mM}$-histidine were $114 \pm 3 \mathrm{Klett}$ units compared to $116 \pm 3$ Klett units for $1.5 \mathrm{~mm}-\mathrm{NH}_{4} \mathrm{Cl}$ and $1 \mathrm{~mm}$-histidine resulted in $210 \pm 12$ Klett units compared to $209 \pm 4 \mathrm{~K}$ lett units for $3 \mathrm{mM}-\mathrm{NH}_{4} \mathrm{Cl}$. Thus, these results confirmed that $P$. putida uses all three nitrogens from histidine and provides a positive control for the growth-yield experiments with $C$. crescentus where only two of the three nitrogens from histidine were utilized.

\section{Activity of enzymes involved in histidine degradation}

In order to learn more about histidine degradation in $C$. crescentus, we attempted to measure the appropriate enzyme activities. Histidase, the first enzyme of the pathway, was found to be induced by either histidine or urocanate (Table 2). Induction was maximal with either 0.4 or $4 \mathrm{~mm}$-histidine, but occurred at a reduced level with urocanate at concentrations up to $4 \mathrm{~mm}$. 


\title{
Table 1. Growth yield with limiting nitrogen sources
}

The final optical density was measured using a Klett-Summerson colorimeter (no. 54 filter). Initial values above 200 Klett units were corrected by dilution of the culture to provide readings within the linear range of optical density.

Nitrogen source

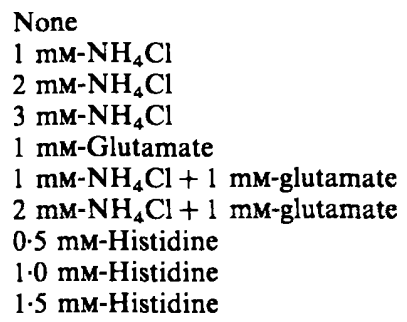

Final optical density

(Klett units, \pm SE)

$$
\begin{gathered}
7 \\
149 \pm 4 \\
305 \pm 11 \\
477 \pm 5 \\
118 \pm 1 \\
313 \pm 7 \\
438 \pm 5 \\
136 \pm 5 \\
320 \pm 4 \\
493 \pm 5
\end{gathered}
$$

\section{Table 2. Histidase induction in complete medium}

\begin{abstract}
Cultures were grown at $33{ }^{\circ} \mathrm{C}$ in $\mathrm{M} 2$ medium containing $1 \mathrm{mM}-\mathrm{NH}_{4} \mathrm{Cl}$ and $0.3 \%$ glucose to a cell density of 50 Klett units (no. 54 filter), inducer was added and growth was allowed to proceed for an additional $3 \mathrm{~h}$. Cultures were incubated with $10 \mu \mathrm{g}$ cetylhexadecyltrimethylammonium bromide $\mathrm{ml}^{-1}$ (CTAB) for $1 \mathrm{~min}$, harvested by centrifugation, washed, and resuspended in $50 \mathrm{~mm}$-potassium phosphate buffer, pH $7 \cdot 5$, containing $5 \mathrm{~mm}-\beta$-mercaptoethanol.
\end{abstract}

$\begin{array}{lc}\begin{array}{c}\text { Addition to } \\ \text { growth medium }\end{array} & \begin{array}{c}\text { Histidase specific activity } \\ \text { [nmol } \mathrm{min}^{-1}\left(\mathrm{OD}_{650} \text { unit }\right)^{-1} \text { ] }\end{array} \\ \text { None } & 0.3 \\ 0.4 \mathrm{mM} \text {-Urocanate } & 1.6 \\ 4 \text { mM-Urocanate } & 3.6 \\ 0.4 \text { mM-Histidine } & 6.0 \\ 4 \text { mM-Histidine } & 6.0\end{array}$

These results could be explained if urocanate is not taken up as well as histidine or if urocanate is a less efficient inducer of histidase synthesis. Both explanations are consistent with the observation that urocanate is not used efficiently as a nitrogen source. However, since the conversion of histidine to urocanate usually is not reversible and since we have found that C. crescentus histidine auxotrophs cannot use urocanate as a source of histidine (data not shown), it is likely that urocanate is an inducer of histidase activity. From the data available, we cannot determine whether histidine also functions as an inducer or whether it must be converted to urocanate which serves as the actual inducer. Urocanate has been shown to be the sole inducer of the expression of histidine utilization genes for most of the Gram-negative bacteria which have been studied (Brill \& Magasanik, 1969; Coote \& Hassall, 1973a, b; Lessie \& Neidhardt, 1967; Schlesinger et al., 1965). In addition to histidase, we were able to demonstrate that histidine caused induction of urocanase and formiminoglutamate iminohydrolase activities (Table 3). Attempts to measure formylglutamate hydrolase were unsuccessful. Thus it is not clear from these results which pathway is utilized.

Wild-type $C$. crescentus exhibits a relatively high affinity, constitutive amino acid uptake activity (Ferber \& Ely, 1982). Since the histidine degradative enzymes are inducible, it was of interest to determine whether histidine uptake activity could be induced as well. Growth in the presence of L-histidine resulted in less than a twofold stimulation of histidine uptake activity (data not shown). In cells where histidine served as the sole nitrogen source, histidine uptake occurred at a slower rate (data not shown). Thus, histidine uptake does not need to be induced for the utilization of histidine as a nitrogen source. 


\title{
Table 3. Levels of histidine degradative enzymes
}

\begin{abstract}
Histidase was assayed as described in Table 2. For the other enzyme assays, cells were grown as described in the legend to Table 2 except that the CTAB step was omitted. Histidine was added at a concentration of $0.4 \mathrm{mM}$. Cell extracts were prepared by ultrasonic disruption and clarified by centrifugation for $15 \mathrm{~min}$ at $24000 \mathrm{~g}$.
\end{abstract}

\begin{tabular}{|c|c|c|c|c|}
\hline \multirow[b]{2}{*}{ Enzyme } & \multirow[b]{2}{*}{$\begin{array}{l}\text { Supplements to } \\
\text { minimal medium }\end{array}$} & \multicolumn{3}{|c|}{ Enzyme activity [nmol $\left.\min ^{-1}(\mathrm{mg} \text { protein })^{-1}\right]$} \\
\hline & & $\begin{array}{l}\text { Glucose } \\
+\mathrm{NH}_{4} \mathrm{Cl}\end{array}$ & $\begin{array}{c}\text { Glucose }+\mathrm{NH}_{4} \mathrm{Cl} \\
+ \text { histidine }\end{array}$ & $\begin{array}{l}\text { Glucose } \\
+ \text { histidine }\end{array}$ \\
\hline $\begin{array}{l}\text { Histidine } \\
\text { Urocanase } \\
\text { Formiminoglutamate }\end{array}$ & & $\begin{array}{c}2 \cdot 6 \\
<1 \\
2 \cdot 9\end{array}$ & $\begin{array}{r}65 \\
9 \\
67\end{array}$ & $\begin{array}{l}97 \\
17 \\
43\end{array}$ \\
\hline
\end{tabular}

iminohydrolase

\section{Waste products of histidine degradation}

To further test the possibility of $C$. crescentus having the four-enzyme pathway, we tested the culture fluid from cells grown on histidine as sole nitrogen source for the presence of formamide (Lund \& Magasanik, 1965). However, no formamide was detected and tests of supernatant from a $10 \%$ trichloroacetic acid precipitation of the culture fluid indicated that only trace amounts of histidine or other nitrogenous compounds were present. Reconstruction experiments demonstrated that formamide in the culture medium is not degraded or taken up by a culture of $C$. crescentus using limiting histidine as sole nitrogen source. Thus, limiting amounts of histidine are taken up from the culture medium quite efficiently by $C$. crescentus and the reduced growth yields are not due to inefficient transport of histidine.

Since formamide was not observed, we tested the culture fluid for the alternative end-product, formate. When cultures were grown on $1 \mathrm{~mm}$-histidine as a nitrogen source, approximately $1 \mathrm{mM}$-formate was observed in the culture fluid by a colorimetric assay, based on the red colour obtained by the reaction of citric or aconitic acids with formic acid (or formate) in the presence of acetic anhydride, propan-2-ol and a small amount of alkali (Lang \& Lang, 1972). In contrast, cultures grown with ammonia as the nitrogen source did not produce detectable levels of formate. Thus, $C$. crescentus appears to degrade histidine by a pathway which yields formate as a waste product. If a pathway similar to that used by the pseudomonads is utilized, 2 mols of ammonia and $1 \mathrm{~mol}$ each of formate and glutamate would be produced per mol of histidine.

\section{Conclusions}

Growth yield experiments with $P$. putida using histidine as sole nitrogen source indicated that this organism uses all three nitrogens efficiently (Table 1; Allen Phillips, personal communication) in accordance with previous observations (Leidgh \& Wheelis, 1973). Thus, if $C$. crescentus degrades histidine using a five-enzyme pathway similar to that of $P$. putida, it is not clear why growth experiments using histidine as sole nitrogen source reflect the use of only two of the three available nitrogens. Certainly, both glutamate and ammonia are used efficiently when presented singly or in combination (Table 1), and histidine can be used as a source of glutamate in mutants unable to use ammonia because they lack glutamate synthase (Ely et al., 1978). Perhaps $C$. crescentus utilizes a novel pathway which results in the production of only 1 mol each of glutamate, formate and ammonia per mol of histidine and the third nitrogen is sequestered as some novel secondary metabolite which we have been unable to detect. Alternatively, it is possible that some form of denitrification of FIGLU may occur with the release of inorganic nitrogen as a waste product.

This research was supported by grant PCM-8003729 from the National Science Foundation to B. E., by a National Research Service Award (GM072331-01) to D.F., and by grant GM34765 from the National Institutes of Health to B.E. We thank Christina Henke for performing the initial experiments with $P$. putida, and Dr Allen Phillips for a critical reading of the manuscript. 


\section{REFERENCES}

Brill, W. J. \& Magasanik, B. (1969). Genetic and metabolic control of histidinase and urocanase in Salmonella typhimurium, strain 15-59. Journal of Biological Chemistry 255, 5392-5402.

Chasin, L. A. \& Magasanik, B. (1968). Induction and repression of the histidine degrading enzymes of Bacillus subtilis. Journal of Biological Chemistry 243, 5165-5178.

Chiaverotti, T. A., Parker, G., Gallant, J. \& Agabian, N. (1981). Conditions which trigger guanosine tetraphosphate accumulation in Caulobacter crescentus. Journal of Bacteriology 145, 1463 1465.

CoOTE, J. G. \& Hassall, H. (1973a). The degradation of L-histidine, imadazolyl-L-lactate and imidazolylpropionate by Pseudomonas testosteroni. Biochemical Journal 132, 404-422.

COOTE, J. G. \& Hassall, H. (1973b). The control of the enzymes degrading histidine and related imidazolyl derivatives in Pseudomonas testosteroni. Biochemical Journal 132, 423-433.

DAvis, R. W., BotsteIN, D. N. \& Roth, J. R. (1980). A Manual for Genetic Engineering: Advanced Bacterial Genetics. Cold Spring Harbor, NY: Cold Spring Harbor Laboratory.

Ely, B., Amarasinghe, A. B. C. \& Bender, R. A. (1978). Ammonia assimilation and glutamate formation in Caulobacter crescentus. Journal of Bacteriology 133, 225-230.

Ferber, D. M. \& Ely, B. (1982). Resistance of amino acid inhibition in Caulobacter crescentus. Molecular and General Genetics 187, 446-452.

Johnson, R. C. \& ELY, B. (1977). Isolation and spontaneously-derived mutants from Caulobacter crescentus. Genetics 86, 25-32.

KURN, N., Shapiro, L. \& Agabian, N. (1977). Effect of carbon source and the role of cyclic adenosine 3',5'-monophosphate on the Caulobacter cell cycle. Journal of Bacteriology 131, 951-959.

LANG, E. \& LANG, H. (1972). Specific colour reaction for the direct identification of formic acid. Zeitschrift für Analytische Chemie 160, 8-10.
LEIDGH, B. J. \& WheEls, M. L. (1973). Genetic control of the histidine dissimilatory pathway in Pseudomonas putida. Molecular and General Genetics 120, 201-210.

Lessie, T. G. \& NeIDHARd, F. C. (1967). Formation and operation of the histidine-degrading pathway in Pseudomonas aeruginosa. Journal of Bacteriology 93, 1800-1810.

LUND, P. \& Magasanik, B. (1965). N-Formimino-Lglutamate formiminohydrolase of Aerobacter aerogenes. Journal of Biological Chemistry 140, 43164319.

MaGasanix, B. (1982). Genetic control of nitrogen assimilation. Annual Review of Genetics 16, 135-168.

Magasanik, B. \& Bowser, H. R. (1955). The degradation of histidine by Aerobacter aerogenes. Journal of Biological Chemistry 213, 571-580.

Meiss, H. K., Brill, W. J. \& Magasanik, B. (1969). Genetic control of histidine degradation in Salmonella typhimurium, strain Lt-2. Journal of Biological Chemistry 244, 5382-5391.

PoINDEXTER, J. S. (1964). Biological properties and classification of the Caulobacter group. Bacteriological Reviews 28, 231-295.

Schlesinger, S., Scotto, P. \& Magasanik, B. (1965). Exogenous and endogenous induction of the histidine-degrading enzymes in Aerobacter aerogenes. Journal of Biological Chemistry 240, 4331-4337.

SMITH, G. R. \& MAGASANIK, B. (1971). Nature and selfregulated synthesis of the repression of the hut operons in Salmonella typhimurium. Proceedings of the National Academy of Sciences of the United States of America 68, 1493-1497.

SMith, G. R., HalPERN, Y. S. \& MaGasanik, B. (1971). Genetic and metabolic control of enzymes responsible for histidine degradation in Salmonella typhimurium. Journal of Biological Chemistry 246, 33203329.

TABOR, H. (1955). Degradation of histidine. In $A$ Symposium on Amino Acid Metabolism, pp. 373-390. Edited by W. D. McElroy \& H. B. Glass. Baltimore : Johns Hopkins Press. 